\title{
Contribución al conocimiento de Stigmaphyllon nudiflorum (Malpighiaceae), una liana endémica en Peligro Crítico y una hipótesis sobre su origen evolutivo en la costa de Ecuador
}

Contribution to the knowledge of Stigmaphyllon nudiflorum

(Malpighiaceae), a critically endangered endemic liana and a hypothesis about the evolutionary origin on the coast of Ecuador

\section{Xavier Cornejo ${ }^{1 *}$}

\begin{abstract}
${ }^{1}$ Herbario GUAY, Departamento de Botánica, Facultad de Ciencias Naturales, Universidad de Guayaquil, Av. Raúl Gómez Lince s.n. y Av. Juan Tanca Marengo (campus Mapasingue), P.O. Box 09-01-10634, Guayaquil, Ecuador.
\end{abstract}

Recibido 3 agosto 2021; revisado 26 agosto 2021; aceptado 20 de diciembre 2021; disponible en línea 30 de diciembre 2021.

\begin{abstract}
Resumen
Se reporta por primera vez para la especie y para el género las alas acrescentes de las sámaras de Stigmaphyllon nudiflorum, una liana de Malpighiaceae endémica del bosque seco de la provincia del Guayas, en la costa de Ecuador. Además, con base a un estudio previo de la edad e historia biogeográfica de este género y en corelación con el origen de los suelos en donde S. nudiflorum habita, se presenta una hipótesis acerca del origen evolutivo de esta y otras especies relacionadas endémicas del área sureste de la cordillera Chongón-Colonche, en el occidente de Ecuador.

Palabras clave: bosque seco, costa, endemismo, Pacífico.
\end{abstract}

\begin{abstract}
The accrescent wings of the sámaras of Stigmaphyllon nudiflorum, a Malpighiaceae liana endemic to the dry forest of the Guayas province, on the coast of Ecuador, are reported here for the first time for the species and for the genus. In addition, based on a previous study of the age and biogeographic history of this genus coupled to the origin of the soils where S. nudiflorum inhabits, a hypothesis is presented here about the evolutionary origin of this and other related species endemic to the southeast area of the Chongón-Colonche mountain range, in western Ecuador.

Key words: coast, dry forest, endemism, Pacífic.
\end{abstract}

\section{Introducción}

Stigmaphyllon A. Juss. es un género monofilético de lianas que pertenece a las Mapighiaceae Juss. (familia de la Ayahuasca), comprende 90 species en el Neotrópico con excepción de S. bannisterioides A. Juss., que también se encuentra en el occidente de África (Anderson, 2011). Trece especies de Stigmaphyllon han sido reportadas en Ecuador (Anderson, 1997; Jorgensen \& León, 1999); entre estas, 3 taxa endémicas y escasamente conocidas habitan bajo los $500 \mathrm{~m}$ en las tierras bajas del bosque seco deciduo del Pacífico Ecuatorial: S. ecuadorense C. Anderson (1993), S. eggersii C. Anderson (1993) y S. nudiflorum Diels (1937). Stigmaphyllon nudiflorum es la única especie en este género que presenta un comportamiento fuertemente caducifolio, esta florece y fructifica únicamente después de descartar sus hojas maduras durante la estación seca; por esta razón, las pocas colecciones fértiles de herbario conocidas de esta especie estrictamente consisten en ramas completamente desfoliadas o produciendo hojas muy jóvenes con flores y escasas veces con unos pocos frutos (Anderson, 1997; obs. pers.). En campo, los individuos de S. nudiflorum se aprecian como alambres desfoliados y poco atractivos de varios metros de longitud, a no ser por la presencia de sus coloridas flores amarillas, de otro modo fácilmente podrían pasar desapercibidos en medio de la vegetación decidua.

Stigmaphyllon nudiflorum fue descrita como una nueva especie con base en el holotipo (Diels 1231, B), colectado el 29 de Septiembre de 1933, $20 \mathrm{~km}$ al oeste de Guayaquil, por el botánico alemán Ludwig Diels mientras estaba en funciones como Director del herbario de Berlín (Diels, 1937; Anderson, 1997); al parecer no existen duplicados (isotipos). La

* Correspondencia del autor:

E-mail: xcornejoguay@gmail.com 
Rev. Cient. Cien. Nat. Ambien. 15(2):299-302

Diciembre 2021
Cornejo • Contribución al conocimiento de

Stigmaphyllon Nudiflorum (Malpighiaceae), una liana endémica en Peligro Crítico y una hipótesis sobre su origen evolutivo en la costa de Ecuador publicación original describe el hábito trepador, las ramas y las flores; de las hojas sólo menciona que no están desarrolladas durante la estación seca y no hay información de los frutos (Diels, 1937). La mencionada colección tipo de $S$. nudiflorum, así como miles de especímenes tipos de plantas provenientes de todas partes del mundo y principalmente de América tropical fueron quemadas en un voraz incendio causado por un bombardeo sorpresivo y al azar (Hugh H. Iltis, com. pers.), realizado por las fuerzas aliadas en la noche entre el 1 al 2 de marzo de 1943, este destruyó el ala izquierda y la porción central del Museo Botánico de Berlín en Dahlem, durante la Segunda Guerra Mundial (Hiepko, 1987). Posteriormente, la designación de un neotipo (Dodson \& Dodson 11199, MO, SEL, proveniente de Capeira, km 21 vía Guayaquil-Daule, en la provincia del Guayas) que consiste en segmentos de ramas, flores y sámaras con un ala desarrollada y una descripción de esta especie han sido presentados en la monografía de este género (Anderson, 1997).

Durante el trabajo de campo realizado en los años 2020 y 2021 para la elaboración de la Flora de Guayaquil (Cornejo, en prep.), se encontraron individuos de Stigmaphyllon nudiflorum en 6 sitios en Cerro Azul, Cerro Blanco y muy cerca de la localidad tipo, estos sitios están localizados en la porción terminal sureste de la cordillera Chongón-Colonche, todos los individuos observados difieren de la descripción de esta especie por presentar frutos con nueces sin alas, nueces crestadas o con alas vestigiales, distintos de los descritos para S. nudiflorum y del neotipo designado (Anderson, 1997). A finales de noviembre de 2021, se observó en campo que frutos con las formas mencionadas son parte de un rango de variabilidad dentro de su desarrollo ontogénico, este no había sido reportado para esta especie y además era desconocido para el género. Este aspecto del ala acrescente de los frutos de $S$. nudiflorum y una hipótesis acerca del posible origen evolutivo de este taxón y de las especies endémicas restantes que habitan en el área de la cordillera Chongón-Colonche y sus alrededores se presenta a continuación.

\section{Materiales y Métodos}

Se estudiaron los especímenes relevantes en montajes o digitalizados en alta resolución de los herbarios GUAY, MICH, y US. Las medidas han sido tomadas de especímenes de herbario y de 9 individuos de Stigmaphyllon nudiflorum estudiados y fotografiados en campo entre febrero de 2019 hasta noviembre de 2021.

\section{Resultados}

Los frutos de Stigmaphyllon nudiflorum: Stigmaphyllon nudiflorum presenta flores con 1 ovario, 3-carpelar, 3-locular; cada flor produce 1-2(-3) nueces, éstas se generan una en cada lóculo (Fig.1: $A, B, C)$. Cada nuez se desarrolla hasta alcanzar 1.4$1.9 \times 1.1-1.2 \mathrm{~cm}$ (en vivo), ca. $13 \times 1 \mathrm{~cm}$ (en seco).

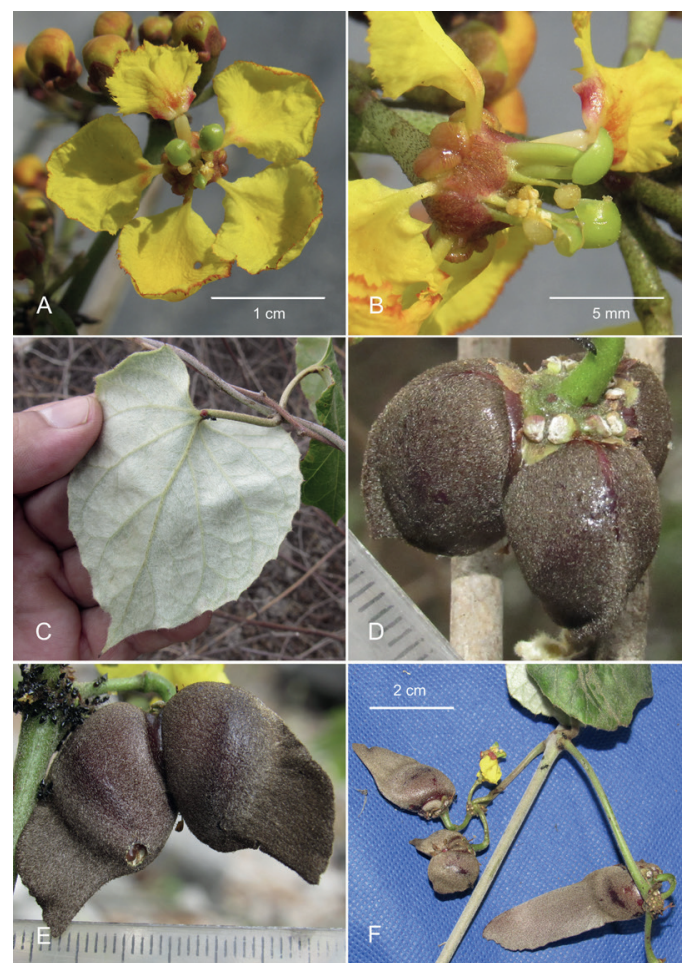

Figura 1. Stigmaphyllon nudiflorum Diels. A, Flor, vista adaxial; B, Acercamiento de la flor, vista lateral; C, Hoja juvenil, envés blanco-tomentoso, vista abaxial; D, Nueces quilladas; $E$, Nueces con alas vestigiales; F, Rama con infructescencias portando frutos en todo el rango de variabilidad, desde nuez crestada (izq.) hasta sámara con ala desarrollada (der.).

Las nueces son lisas, de color café-oscuro a caférojizas, cubiertas por una abundante pubescencia corta; inicialmente alcanzan su mayor crecimiento sin tener un ala desarrollada, más bien son quilladas o con una cresta dorsal de 2-3 mm (Fig. 1:D), o con alas vestigiales ca. $1.1 \times 1.3 \mathrm{~cm}$ (Fig. $1: \mathrm{E}$ ), hasta este grado de desarrollo se encuentran mientras aún hay flores en la misma inflorescencia. Posteriormente, las alas de los frutos se tornan acrescentes, es decir, presentan un crecimiento mayor después del desarrollo de la nuez hasta conformar verdaderas sámaras oblongas, dorsalmente 1-aladas, de color café oscuro, cuyas dimensiones en vivo son 3.6-4.5 x 1-1.2 cm (Fig. $1: F)$. El ala de la sámara es suave, membranosa y más flexible hacia la porción distal, siendo en cuanto a la textura en fresco similar a la cola de un pez. Estas observaciones de campo permitieron conocer la naturaleza acrescente en la ontogenia de los frutos de S. nudiflorum, esta característica era desconocida para esta especie y género, respectivamente.

Frutos con el ala dorsal reducida a una cresta o quilla es un carácter distintivo presente solamente en tres especies en el Neotrópico, estas son: $S$. bannisterioides (L.) C.E. Anderson distribuida desde Mesomerica hasta Brazil y en las Indias Occidentales, S. harleyi W.R. Anderson restringida a Bahia, 
Rev. Cient. Cien. Nat. Ambien. 15(2):299-302 Diciembre 2021
Cornejo • Contribución al conocimiento de Stigmaphyllon Nudiflorum (Malpighiaceae), una liana endémica en Peligro Crítico y una hipótesis sobre su origen evolutivo en la costa de Ecuador
Brasil, y S. paralias del este de Brazil (op. cit.); sin embargo, el ala vestigial en estas especies al parecer permanece constante y no presentan un desarrollo acrescente hasta llegar a ser una sámara, como sucede en $S$. nudiflorum. La presencia de especies en este género con frutos quillados o crestados o con alas vestigiales o reducidas adaptadas para dispersión por agua, como lo es también Tetrapteris subaptera Cuatrec. (Malpighiaceae) que habita en los manglares del Pacífico desde el sureste de Panamá hasta el noroccidente de Ecuador (Cornejo, 2014), inicialmente condujo a pensar que las poblaciones de S. nudiflorum que presentaban frutos similares podrían tratarse de una especie no descrita. Finalmente, el 27 de noviembre de 2021, esto es, al final del quinto mes del rango total del periodo de frutificación de esta especie se encontró en La Artillería, en la provincia del Guayas, 3 individuos de S. nudiflorum exhibiendo los frutos en toda la gama del desarrollo ontogénico, esto es, desde nueces desarrolladas y quilladas hasta sámaras unialadas con alas distintivas y bien desarrolladas (Fig. 1:F), comprobándose que el ala es un elemento acrescente en el fruto, es decir, no crece hasta su talla máxima conjuntamente con la formación de la nuez, sino que su mayor crecimiento es posterior al desarrollo de ésta.

Hábitat, distribución:- Stigmaphyllon nudiflorum habita en los fragmentos secundarios de bosque seco deciduo del Pacífico ecuatorial, y es ocasionalmente persistente en áreas alteradas con especies nativas remanentes en ambientes que reciben un fuerte impacto de la radiación solar, entre 20 hasta $200 \mathrm{~m}$. Crece en suelos rocosos, calizos, con buen drenaje, localizados hacia la porción terminal sureste de la cordillera Chongón-Colonche, al oeste y hacia norte de la ciudad de Guayaquil, en la provincia del Guayas, en la costa de Ecuador (Anderson, 1997).

Estado de Conservation: Esta especie no se encuentra dentro del Sistema Nacional de Áreas Protegidas del Ecuador y ha sido categorizada En Peligro Crítico, CR B2ab(iii) (Barriga en León-Yánez et al., 2011). Aunque se encuentra parcialmente protegida en reservas privadas como el Bosque Protector Cerro Blanco y en el Bosque La Prosperina que es manejado por la ESPOL, sus principales amenazas son la deforestación, la ampliación de la frontera urbana de la ciudad de Guayaquil y las quemas provocadas y sin control de la vegetación que se producen todos los años durante la estación seca.

Fenología: Stigmaphyllon nudiflorum florece y fructifica mayormente durante la estación seca cuando las plantas están deciduas o generando hojas jóvenes. Las flores se producen desde julio hasta enero y fructifica desde agosto hasta febrero; la producción de sámaras maduras completamente desarrolladas coincide con el inicio de la estación lluviosa, durante este periodo se produce la germinación y crecimiento vegetativo con la producción de tallos, ramas y hojas. Las nuevas hojas se desarrollan y alcanzan la madurez desde finales de diciembre hasta mayo, mayormente durante la temporada de lluvias, éstas caen antes de la floración.

El comportamiento fuertemente caducifolio y los frutos quillados, crestados, de lo contrario con alas muy reducidas, es una combinación única de características que permiten reconocer fácilmente a esta especie. Stigmaphyllon nudiflorum se ha pasado por alto debido a la fenología completamente decidua, con los tallos sin hojas que permanecen como alambres poco atractivos y los frutos pequeños de color marrón oscuro que se pueden confundir fácilmente entre la vegetación nativa leñosa de hoja caduca de color marrón claro a grisáceo.

Interacciones biológicas: Stigmaphyllon nudiflorum es una liana mirmecófila, al menos dos especies de hormigas han sido observadas visitando activamente las plantas de esta especie, una en los nectarios de las inflorescencias, otras formando grupos numerosos en los nudos alrededor de la base en donde se inserta el pedúnculo de la inflorescencia. Además, las flores poseen elaióforos en la base externa de los sépalos, éstas son glándulas especializadas en la secreción de aceites apetecidos por varias especies de abejas nativas del género Centris, como también sucede en otras lianas de Malpighiaceae en el bosque seco deciduo del Pacífico Ecuatorial (BSDPE) que poseen flores amarillas, como Tetrapterys jamesonii Turcz. (obs. pers.).

Hipótesis del origen de las especies de Stigmaphyllon endémicas del occidente de Ecuador: Considerando la edad e historia biogeográfica de Stigmaphyllon y la formación de los suelos en donde habita $S$. nudiflorum se presenta una hipótesis sobre el posible origen evolutivo de esta especie y de otras especies endémicas relacionadas presentes en el área de la cordillera Chongón-Colonche y sus alrededores. La reconstrucción de la edad e historia biogeográfica de Stigmaphyllon basada en una región nuclear de ADN (PHYC) y dos regiones de ADN ribosomal (ETS, ITS) utilizando métodos de parsimonia y análisis Bayesianos, indica que el ancestro común más reciente en este género se originó en los bosques lluviosos del Atlántico/región de la Caatinga en el noreste de Brasil hace ca. 26 millones de años; Stigmaphyllon mayormente se diversificó ca. 26.5 hasta 21 millones de años y colonizó la amazonía en dos eventos que se realizaron entre 22 a 6 millones de años (de Almeida y van den Berg 2020; Willis et al., 2014). La evidencia fósil indica que los suelos donde Stigmaphyllon nudiflorum habita estuvieron sumergidos como un área arrecifal subsuperficial en un ecosistema marino abierto, estos suelos emergieron como consecuencia de la colisión de la costa de Ecuador contra las márgenes occidentales de los Andes, probablemente durante o después del Oligoceno tardío-Mioceno (ca. 28.1 hasta 20.4 millones de años; Jaillard et al., 1995; Moreira, 2019). Las nuevas tierras emergentes de la proto-cordillera Chongón-Colonche inicialmente fueron islas que permitieron la colonización de especies de tierra firme y la formación de antiguos 
Rev. Cient. Cien. Nat. Ambien. 15(2):299-302

Diciembre 2021
Cornejo • Contribución al conocimiento de

Stigmaphyllon Nudiflorum (Malpighiaceae), una liana endémica en Peligro Crítico y una hipótesis sobre su origen evolutivo en la costa de Ecuador ecosistemas terrestres en el occidente de Ecuador, en los que se habrían producido procesos de especiación generando un número de especies endémicas locales y regionales (Wolf, 1892; Cornejo \& Lombardi, 2021).

Consecuentemente, con base en la reconstrucción de la edad e historia biogeográfica del género (op cit.), corelacionada con la evidencia fósil y las fechas del origen de los suelos en la cordillera Chongón-Colonche en el occidente de Ecuador, más los eventos tectónicos regionales, la morfología del fruto y su síndrome de dispersión tipo anemocoria, aquí se hipotetiza que la llegada del género Stigmaphyllon al occidente de Ecuador se produjo por vientos que soplaban desde la Amazonía en dirección hacia el oeste y que la formación de los lineajes de las conespecíficas endémicas de los bosques secos del occidente de Ecuador como S. nudiflorum, S. ecuadorense, S. eggersii, y también de Amorimia kariniana W.R. Anderson y Mascagnia haenkeana W.R. Anderson (Malpighiaceae), ambas endémicas locales del sureste de la cordillera Chongón-Colonche y de la provincia del Guayas, con frutos samaroides, cuyo patrón de distribución es similar al de S. nudiflorum, fueron el resultado de eventos vicariantes que se habrían producido después de cruzar los Andes hacia el Pacífico por parte de las especies anemófilas ancestrales durante el Neogeno (23 hasta 2.58 millones de años).

\section{Especímenes estudiados}

ECUADOR. Guayas: Capeira, km 21 Guayaquil to Daule, 201'S, 7957'W, 20-180 m, 13 Sep 1981 (fr), C.H. Dodson \& P.M. Dodson 11199 (MO, foto en MICH!, neotipo); $22 \mathrm{~km} \mathrm{~N}$ of Guayaquil in road to Daule, $2^{\circ} 00^{\prime} \mathrm{S}$, 7958'W, 20-150 m, 14 Jul 1986 (fl, fr), A. Gentry \& C.H. Dodson 54823 (MICH!). Road from Guayaquil to Quevedo, km 78, ca. $1^{\circ} 24^{\prime} \mathrm{S}, 7^{\circ} 26^{\prime} \mathrm{W}, 100 \mathrm{~m}, 12 \mathrm{Nov}$ 1961 (fr), C.H. Dodson \& L.B. Thien 1295 (US!). 9 km N of Guayaquil on road to Daule, 50 m, 24 Sep 1961 (fl), C.H. Dodson \& L.B. Thien 712 (US!). Bosque Protector Cerro Blanco, 209's, 7959'W, 100 m, Sep 2021 (fl, fr), X. Cornejo \& J. Josse 9392 (GUAY!); Cerro Azul, $2^{\circ} 10^{\prime} \mathrm{S}, 79^{\circ} 58^{\prime} \mathrm{W}, 100 \mathrm{~m}$, Sep 2021 (fl, fr), X. Cornejo \& J. Josse s.n. (GUAY!). Chongoncito, ca. $25 \mathrm{~km}$ W from Guayaquil, 2¹4'S 8004'W, 20 m, 17 Sep 2021 (fl, fr), X. Cornejo, C. Calderón \& J. Rambay 9393 (GUAY!).

\section{Agradecimientos}

El autor expresa su agradecimiento a Christiane Anderson $(\mathrm{MICH})$ por sus útiles comentarios de las especies de Stigmaphyllon y por proveer al autor de la literatura pertinente y de imágenes digitales de las colecciones de $\mathrm{S}$. nudiflorum de $\mathrm{MICH}$, y en especial del neotipo. Al Ing. Jorge Josse Moncayo, con quien ha realizado las salidas de campo a Cerro Azul y Cerro Blanco durante los años 2020 y 2021, que permitieron encontrar a esta especie y notar in situ su característica de generar frutos sin alas desarrolladas en los estadíos iniciales.

\section{Referencias}

Anderson, C.E. 1997. Monograph of Stigmaphyllon (Malpighiaceae). Systematic Botany Monographs 51: 1-313.

Barriga, P. 2017. Stigmaphyllon nudiflorum. En: LeónYánez, S., R. Valencia, N. Pitman, L. Endara, C. Ulloa Ulloa y H. Navarrete (Eds). Libro Rojo de Plantas Endémicas del Ecuador. Publicaciones del Herbario QCA, Pontificia Universidad Católica del Ecuador, Quito. <https://bioweb. bio/floraweb/librorojo/FichaEspecie/Stigmaphyllon\%20 nudiflorum>, acceso jueves, 23 de diciembre de 2021.

Cornejo, X. 2014. Plants of the South American Pacific Mangrove swamps (Colombia, Ecuador, Perú). Ed. EDUQUIL. $312 \mathrm{p}$.

Cornejo, X. and Lombardi, J.A. 2021. Salacia juradoi (Celastraceae), a new species from coastal Ecuador. Phytotaxa, 524(2): 125-130.

de Almeida, R.F. and van den Berg, C. 2020. Biogeography of Stigmaphyllon (Malpighiaceae) and a meta-analysis of vascular plant lineages diversified in the Brazilian Atlantic Rainforests point to the Late Eocene origins of this megadiverse biome. Plants, 9(11), p.1569.

Diels, L. 1937. Beiträge zur Kenntnis der Vegetation und Flora von Ecuador. Bibliotheca Botanica. 29(116): 1-190. Stuttgart.

Hiepko, P. 1987. The collections of the Botanical Museum Berlin-Dahlem (B) and their history. Englera 7: 219-252.

Jaillard, É., Ordoñez, M., Benitez, S., Berrones, G., Jiménez, N., Montenegro, G. y Zambrano, I. 1995. Basin development in an Accretionary, Oceanic-floored Fore-Arc Setting: Southem Coastal Ecuador during Late CretaceousLate Eocene Time. In A. J. Tankard, R. Suárez S., and H. J. Welsink (Eds.), Petroleum Basins of South America, American Association of Petroleum Geologist Memoir 62: 615-631.

Jørgensen, P. M. y S. León-Yánez, eds. 1999. Catalogue of the Vascular Plants of Ecuador. Monogr. Syst. Bot. Missouri Bot. Gard. 75: 1-1181.

Moreira, R.C. 2019. Bioestratigrafía y ambiente sedimentario de depositación de las calizas de la formación San Eduardo (Eoceno) al sur del Bosque Protector Cerro Blanco, Provincia del Guayas. Trabajo de titulación previo a la obtención del grado académico de Ingeniero Geólogo. Facultad de Ciencias Naturales, Universidad de Guayaquil, $97 \mathrm{pp}$.

Willis, C.G., Franzone, B.F., Xi, Z., Davis, C.C. 2014. The establishment of Central American migratory corridors and the biogeographic origins of seasonally dry tropical forests in Mexico. Front. Genet. 2014, 5, 433.

Wolf, T. 1892. Geografía y Geología del Ecuador. Editorial Casa de la Cultura Ecuatoriana, Quito, Ecuador, p. 798. 